\title{
DIFFUSION, WINDS AND X-RAYS FROM MAGNETIC STARS
}

\author{
J. BABEL \\ Switzerland, (babeljr@bluewin.ch $)^{\dagger}$
}

\begin{abstract}
We propose a self-consistent approach of the CP star phenomenon and try to solve simultaneously the problems of abundance spots, wind and X-ray emission from these stars. We also discuss the periodic $\mathrm{X}$-ray emission from the $\mathrm{O} 7 \mathrm{~V}$ star $\theta^{1}$ Orionis $\mathrm{C}$ and its link with Bp stars.
\end{abstract}

\section{Introduction}

Despite many attempts of modelling, the building of abundance spots on the surface of magnetic Cp stars and their locus is still not explained. Furthermore, processes like strong mass loss and diffusion seem to operate simultaneously while we expect them to exclude each other (e.g. Michaud 1986). The recent observation of X-ray emission from magnetic CP stars (Drake et al. 1994, Babel \& Montmerle 1997a; hereafter BM), permits perhaps to derive the best constraints on the mass loss from these objects, and force us to investigate simultaneously the onset of winds on these stars, $\mathrm{X}$-rays and diffusion processes.

\section{The wind from $\mathrm{CP}$ stars}

Previous wind studies based on Sobolev approximation (Abbott 1979, CAK) lead to the conclusion that it was possible to drive a strong wind from main sequence CP stars as soon as $T_{e f f} \gtrsim 14000$ $K$. This result was in contradiction with diffusion models which required very weak winds. The inclusion of the very detailed line-blocking and photospheric self-shadowing of the wind driving lines permitted to redefine the wind limits and to improve much the agreement with diffusion predictions (Babel 1996). We could then separate the HR-diagram in 3 zones: a "static hydrogen" zone with $\dot{M} \lesssim 10^{-15} \mathrm{M}_{\odot} \mathrm{yr}^{-1}$, a "multicomponent wind" zone and an "homogeneous wind" zone. Ap SrCrEu, Ap Si, HgMn and ${ }^{3} \mathrm{He}$ stars are currently found in the first zone, while He-rich stars in the second and third zones, in a zone where radiatively driven winds fade.

\section{X-ray emission from a magnetically confined wind-shock}

A very stimulating result from ROSAT is that magnetic CP stars are intrinsic X-ray emittors (Drake et al. 1994, BM). The X-ray luminosity and temperature, the known magnetic field and theoretical mass loss rates from the best observed case IQ Aur (AO star) lead us to propose a new model. In the MCWS model, the radiatively driven winds from the two magnetic hemispheres are confined towards the equator where they collide, lead to a strong shock and X-ray emission. We peform a complete modelling of these processes including the radiation driven wind and the cooling postshock region in a dipolar magnetic field geometry. The model explains both the X-ray luminosity and X-ray temperature from IQ Aur. A very interesting prediction of the model is the presence of a corotating high density cooling disk. This disk, which is in pressure balance with the ram pressure of the wind, opens easily the magnetic field lines in presence of centrifugal forces. For IQ Aur, $r_{A}^{D} \simeq 3 R_{*}$ while the Alfvén radius for the wind alone is around $40 R_{*}$ !

If no feedback is assumed between chemical anomalies and the onset of wind on IQ Aur, the mass

'Contact address: Bundesamt für Statistik, Sekt. Hochschule und Wissenschaft, Holzikofenweg 8, 3003 Bern, Switzerland 
loss rate which permits to explain the X-ray observation $\left(10^{-10} \mathrm{M}_{\odot} \mathrm{yr}^{-1}\right)$ is inconsistent with diffusion models. Babel (1996), however, obtained that CP stars are very close to the wind limits and that this limit highly depends on the chemical composition at the wind base. This suggests that chemical overabundances might be the cause of the occurence of a strong wind on IQ Aur. We thus expect transient phases of mass loss on CP stars with typically a few percents of CP stars being in the wind (and thus $\mathrm{X}$-ray emission) phase (BM).

\section{On the periodic X-ray emission from $\theta^{1}$ Ori $\mathrm{C}$}

The very hot 07V star presents a strong periodic variation of UV and optical lines (e.g. Stahl et al. 1996). These authors suggested that the variations are caused by the presence of a dipolar magnetic field embedded in the wind from $\theta^{1}$ Ori $\mathrm{C}$ and by an oblique magnetic rotator. More recently, Gagné et al. (1997) discovered a strong periodic variation of the ROSAT HRI count rate from this star. This variation seemed to reinforce the hypothesis of a magnetic origin. Assuming a dipolar magnetic geometry, we model the wind, postshock region and $\mathrm{X}$-ray emission from $\theta^{1}$ Ori $\mathrm{C}$ (Babel \& Montmerle 1997b). We compute the X-ray emission spectra from each point of the magnetosphere, the 3-D absorption by the surrounding "cold" wind, and convolve with the detector effective area. We obtain that the observed X-ray luminosity is explained by the MCWS model provided that a dipolar magnetic field with $B_{d}=270-370 \mathrm{G}$ (strength at the pole for $r=R_{*}$ ) is present. The observed periodic variability is well explained by an oblique rotator model and by eclipses caused by the cooling disk and the star, and by the varying absorption by the "cold" wind. $\theta^{1}$ Ori $\mathrm{C}$ may thus be the hottest analog to $\mathrm{Bp}$ stars and a promising target for magnetic field measurements or high resolution X-ray spectroscopy.

\section{Mass loss spots and magnetic fields}

The photospheric shadowing of the wind driving lines makes the radiative acceleration depends much on the Doppler shift and thus on the projected wind velocity. Furthermore, the mass loss rate is highly dependent on the forces at the sonic point. In the presence of a strong magnetic field, the wind follows the magnetic field lines, so that the projected velocity is reduced, the radiative acceleration is reduced and the wind might fade. The detailed effect, computed in 3-D radiative transfer (Babel in preparation), shows that the effect is very large though the angle dependence is much slowlier than for a simple projection of the wind velocity. Photospheric shadowing thus leads easily to mass loss (and thus abundance) spots at the magnetic poles (BM, see also Babel 1992). For an oblique magnetic rotator, the situation is even more interesting due to the combination of radiative acceleration and centrifugal forces along magnetic field lines. Numerical simulations (Babel in preparation) for a magnetic dipole show that even a moderate rotation velocity (i.e. $v_{e q} \simeq 100 \mathrm{~km} / \mathrm{s}$ ) could make the mass loss spots move out of the magnetic pole (easily by $30^{\circ}$ ) and, depending on $\beta$, have various shapes like: ring, lunated or elongated spots, etc.. These shapes are in much better agreement with structures currently determined by Doppler Imaging and may also explain the location of the helium spots on $\sigma$ Ori E (e.g. Groote \& Hunger 1996).

\section{References}

Abbott, D.C. (1979), in IAU Symp. 83, Mass loss and Evolution of O-type stars, ed. P.S. Conti and C. de Loorre, (Dordrecht: Reidel), p. 237

Babel, J. (1992), $A \mathscr{E A} 258,449$

Babel, J. (1996), A\&A 309,867

Babel, J., Montmerle, T. (1997a), $A \& A 323,121$

Babel, J., Montmerle, T. (1997b), Apj 485, L29

Drake, S.A., et al. (1994), Apj 420,387

Gagné, M., et al. (1997), Apj 478, L87

Groote, D., Hunger, K. (1996), 319, 250

Michaud, G. (1986), in Hydrogen deficient stars and related objects, Eds. K. Hunger et al., D. Reidel, p. 453

Stahl, O, et al. (1996), A\&A 312,539 